\title{
La muséologie à la croisée des chemins
}

\section{La museología en la encrucijada de los caminos}

\author{
François MAIRESSE \\ Université de Paris 3, Cerlis, Labex ICCAv \\ francois.mairesse@univ-paris3.fr
}

Recibido: 4-07-2014

Aceptado: 3-11-2014

\begin{abstract}
RÉsumÉ
Ces dernières années, un grand nombre de publications sur les perspectives d'évolution du monde des musées ont été publiées. La réflexion sur le devenir de ces institutions n'est pas pour autant récente: la prospective, cette démarche visant à élaborer de possibles scénarios sur l'avenir, n'est pas à proprement parler muséologique, mais elle intéresse forcément le muséologue s'interrogeant sur le champ muséal. A vrai dire, ce sont surtout des professionnels de musée qui ont écrit sur cette question (dans le contexte muséal), afin d'adapter l'institution aux nouveaux défis. Quelques muséologues se sont cependant risqués à cet exercice, de manière plus ou moins heureuse. Cet article a pour ambition, après avoir présenté la littérature prospective sur le musée depuis ces cinquante dernières années et analysé les résultats récents des rapports de prospective, de s'interroger sur l'apport spécifique d'une réflexion muséologique quant au futur des musées, par-delà les axes classiques (démographie, économie, nouvelles technologies) qui sont le plus souvent retenus.
\end{abstract}

Mots cLÉs: Muséologie. Avenir des musées. Prospective muséale. Centre pour l'avenir des musées.

\section{RESUMEN}

Durante los últimos años han aparecido un gran número de publicaciones sobre las perspectivas de evolución del mundo de los museos. La reflexión sobre el futuro de estas instituciones no es reciente: la prospectiva, método dirigido a elaborar posibles escenarios en el futuro, no es estrictamente hablando museológica, pero necesariamente interesa al museólogo interrogarse sobre el campo museal. A decir verdad, en su mayoría son profesionales del museo quienes han escrito sobre el tema (en el contexto museal) para adaptar la institución a los nuevos retos. Algunos museólogos, se han arriesgado a este ejercicio, de manera más o menos afortunada. El artículo pretende, después de presentar la literatura prospectiva sobre el museo durante los últimos cincuenta años, y de analizar los recientes resultados de los informes de prospectiva, interrogarse sobre la contribución especifica de una reflexión museológica acerca del futuro de los museos más allá de los ejes clásicos (demografia, economía, nuevas tecnologías) que se utilizan con más frecuencia.

Palabras Clave: Museología. Futuro de los Museos. Prospectiva museal. Centro para el Futuro de los Museos. 


\section{Captatio benevolentiæ}

La formule remonte à l'époque de la rhétorique antique: l'orateur, au début de son allocution, cherche à susciter l'intérêt et la bienveillance de son auditoire. La muséologie et les musées sont confrontés à un problème similaire. Durant trois décennies, le phénomène muséal a suscité un intérêt grandissant, induisant la création d'un très grand nombre d'édifices, tandis que les formations en muséologie ou museum studies se multipliaient à travers le monde. La crise économique est-elle sur le point d'inverser ce phénomène? A tout le moins, le monde des musées s'interroge actuellement sur son futur: des transformations semblent nécessaires, en témoignent les très nombreuses interrogations sur le futur de l'institution, relayées notamment par les associations de musée.

Ce questionnement ne peut manquer de se répercuter sur les études muséales ou la muséologie. De quelque manière qu'on puisse la définir, l'histoire de cette discipline n'est pas très ancienne. Certes, il existe bien un certain nombre d'incunables liés au domaine, à commencer par les Inscriptiones de Samuel Quiccheberg (1565), mais c'est essentiellement à la fin du XIX ${ }^{\mathrm{e}}$ siècle, à partir de la constitution de la profession des conservateurs et chercheurs des musées, qu'émerge progressivement ce champ particulier de recherches (Lorente 2012a; Mairesse y Desvallées 2011). Au départ centrée sur les questions de description, de classement ou de taxinomie, la muséologie s'interroge progressivement sur le dispositif muséal puis, de manière plus générale, sur son organisation et son histoire. Longtemps, c'est le terme de «muséographie » qui, en français, sera utilisé pour définir ce champ de recherche et d'activités. Au sortir de la Seconde guerre mondiale, c'est cependant le mot « muséologie » qui s'impose, notamment à travers la définition classique qu'en donne Georges Henri Rivière, alors directeur du Conseil international des musées (ICOM) (Rivière, 1989). A l'époque, la notoriété de Rivière donne une dimension internationale à cette définition, qui sera largement reprise et continue d'être utilisée un peu partout dans le monde, a fortiori dans la francophonie, dans les pays méditerranéens et en Amérique latine.

Dès cette époque, deux voies de recherche relativement différentes se distinguent, qui vont être renforcées par le clivage linguistique au sein du monde académique et, notamment, par la pro- gressive suprématie de la langue anglaise. A partir des années 1970, le courant de la muséologie scientifique, rassemblant des penseurs venus de l'Est (Zbyněk Stránský, Anna Grégorová, Klaus Schreiner ou plus tard Ivo Maroévić), cherche à détacher la muséologie du musée, en présentant son objet de recherche comme «l'étude d'un rapport spécifique de l'homme à la réalité». De manière générale cependant, l'enseignement de la muséologie reste essentiellement lié à la figure du musée dont il présente les modes de fonctionnement et pour lesquelles il tente de préparer les étudiants à s'intégrer au sein de la profession muséale. La domination progressive de l'anglais dans les échanges internationaux a par ailleurs renforcé le caractère relativement pragmatique de la formation. Au niveau mondial, la production académique adopte de plus en plus cette langue, mais aussi ses références, à commencer par le vocable museum studies, qui est généralement préféré à celui de muséologie dans les pays anglo-saxons.

Si le futur des musées apparaît comme difficile à prévoir, qu'en est-il de la muséologie? Est-elle toujours capable de capter l'intérêt des générations futures? Son enseignement et sa recherche sont-ils adaptés au développement futur des musées, tels qu'ils sont actuellement envisagés? Je souhaiterais, dans cet article, partir de cette littérature prospective et des tendances qu'elle dégage au niveau du musée dans les prochaines années, pour m'interroger dans un second temps sur la muséologie elle-même, son rôle et sa situation actuelle, mais aussi son avenir.

\section{Crise et recherches prospectives dans le secteur muséal}

Les musées de nombreux pays occidentaux sont largement touchés, depuis environ cinq années, par le ralentissement de l'activité économique, voire la récession et par les mesures d'économies qui ont été prises afin de réduire les déficits publics ${ }^{1}$. C'est dans un tel contexte qu'ont été publiés plusieurs rapports, essentiellement anglo-saxons, explorant le futur des musées. J'évoquerai ici les résultats présentés dans six rapports ou ouvrages à mon sens assez représentatifs de ce type de littérature : trois ont été rédigés par le Center for the future of Museums appartenant à l'American Alliance of Museums. Il s'agit du premier des documents par lequel le centre a inauguré en 2008 sa politique éditoriale, Museums and society 2034: 
trends and potential future, ainsi que de deux analyses plus récentes intitulées Trendswatch 2012 et Trendswatch 2013, décrivant chacun un certain nombre de tendances et facteurs supposés influencer le monde des musées dans l'avenir $^{2}$. L'association hollandaise des musées, quant à elle, s'est penchée sur l'avenir de son secteur avec l'Agenda 2026. Study on the Future of the Dutch Museum Sector. L'un des derniers rapports en date a été produit par la Museums Association: le rapport Museums 2020, présentant le possible avenir des musées britanniques, a été soumis à un large processus de discussion au sein de la communauté muséale ${ }^{3}$. Enfin, un dernier ouvrage, rédigé par Peter et Léontine van Mensch, analyse d'un point de vue plus théorique les nouvelles tendances dans le domaine de la muséologie et des musées (Mensch 2011). Bien sûr, chaque pays présente des particularités: les Etats-Unis insistent sur leur population multiethnique, les Pays-Bas évoquent leur conurbation (le Randstad) ainsi que l'influence de l'Union européenne, tandis que la Grande Bretagne se penche sur son système de financement ${ }^{4}$. Il n'en demeure pas moins que si aucun de ces rapports ne prétend à une vision universelle, leur lecture conjointe permet de faire apparaître un certain nombre de récurrences.

\section{Quelles évolutions du musée pour le futur?}

Trois types de transformations sont particulièrement mis en lumière: la retraite des boomers, la récession et le crowdsourcing. La mise à la retraite progressive de la génération du baby boom, née juste après la Seconde guerre mondiale, constitue en effet un changement attendu. Cette génération, qui a connu les années de croissance de l'après-guerre, bénéficie d'un pouvoir d'achat important et d'une espérance de vie supérieure à celle des générations précédentes. Plusieurs auteurs se réjouissent de l'augmentation du temps libre dont disposeront ces nouveaux retraités qui pourraient s'investir dans le bénévolat ou fréquenter assidûment les musées, mais il n'est pas impossible que ces derniers se tournent vers d'autres projets qui leur sont également accessibles, par exemple les voyages à l'étranger. Si cette génération dispose d'une plus grande espérance de vie et d'un bon pouvoir d'achat, il n'en reste pas moins que les musées vont devoir s'adapter à de potentiels visiteurs plus âgés (et exigeants).
La crise économique constitue un autre élément régulièrement évoqué. De manière spontanée, la plupart des rapports -autant aux ÉtatsUnis qu'en Grande Bretagne ou aux Pays-Basconsidèrent la baisse structurelle des subventions publiques comme acquise. Par ailleurs, suggèrent plusieurs auteurs, le risque de persistance de la crise économique induira une diminution du pouvoir d'achat d'une partie de la population, et probablement l'augmentation des écarts de revenus au sein de la société. Dans un tel contexte, les musées doivent se mettre à la recherche de nouveaux modèles économiques, reposant moins sur les pouvoirs publics, sans pour autant s'appuyer sur une logique trop commerciale. L'une des solutions les plus régulièrement présentées est liée aux nouvelles technologies, à la révolution 2.0 et au musée participatif. Cette logique, qui a fait l'objet de nombreux articles et ouvrages, touche aussi bien au financement par la foule (crowdfunding) qu'au travail bénévole et à la participation de la population dans la gestion muséale (crowdsourcing), transformant, en quelque sorte, les rapports de production et la production ellemême, soit le contenu du musée et l'éthique de son fonctionnement.

\section{Une tendance qui s'impose?}

Mis à part l'ouvrage des époux van Mensch, les rapports insistent peu sur l'évolution interne du secteur muséal lui-même, notamment pour ce qui concerne les questions liées aux collections, et par exemple les nouvelles manières dont cellesci pourraient être utilisées dans les années à venir. Le principe de la mobilité des collections, visant à mieux les faire circuler et ainsi favoriser leur utilisation, avait dès fait l'objet de nombreuses études dès le début des années 2000 (Pettersson et al. 2010). Le lien entre les objets et les technologies numériques ne passe pas non plus seulement par leur dématérialisation, mais aussi par l'Internet des objets (souvent présenté, à l'instar du web sémantique, comme une troisième révolution de la toile).

En revanche, et sans doute en dehors des très grands musées superstars (Frey y Meier 2006), la tendance principale du monde muséal qui semble s'imposer repose sur ce lien de plus en plus fort entre le musée et sa communauté - ou plus précisément la community (dans le sens des parties prenantes ou stakeholders, intégrant publics et non-publics, spécialistes, journalistes 
locaux, autres lieux de conservation ou de recherche, etc.). La relation entre le musée et son territoire est ancienne, on la retrouve par exemple déjà dans les travaux de Brown Goode, à la fin du XIX $^{\mathrm{e}}$ siècle (Brown Goode 1896). Pendant longtemps, pourrait-on dire, c'est cependant plutôt l'adaptation du musée à l'économie de marché qui avait été présentée comme primordiale, le modèle de Bilbao constituant, en quelque sorte, l'un des jalons forts de cette logique économique. La sortie d'ouvrages sur le travail social du musée ou sur les questions d'inclusions sociale, montre une tendance que certains auteurs, comme Black (2012), évoquent comme la plus importante pour les années à venir. Le rôle social des musées constitue lui aussi un fait ancien dont les prémices remontent au moins au $\mathrm{XIX}^{\mathrm{e}}$ siècle. Ce phénomène a connu des évolutions importantes, et s'il a notamment été en vogue durant les années 1970-80, à travers la nouvelle muséologie en France ou le concept de musée de voisinage aux Etats-Unis, il avait connu un succès nettement plus relatif dans les décennies qui suivirent, alors que le spectaculaire muséal s'imposait au gré des nouvelles réalisations architecturales et de l'engouement pour les grandes expositions temporaires. C'est pourtant une sorte de mouvement cyclique qu'il convient de reconnaître dans l'évolution actuelle, alors que la question $\mathrm{du}$ lien social ressurgit de plus belle au début du $\mathrm{XXI}^{\mathrm{e}}$ siècle, notamment à travers les réflexions de Richard Sandell sur l'inclusion sociale (Sandel 1998, 2002). Quoi qu'il en soit, et mis à part, sans doute, les musées superstars, l'avenir muséal semble essentiellement passer par le chemin du social et du lien avec les communautés.

\section{Quelle approche de la muséologie pour demain?}

Qu'elle se présente comme une activité scientifique indépendante ou comme une pratique, la muséologie se développe à travers les mouvements de transformation de l'institution muséale. Ainsi, la nouvelle muséologie, initiée à l'aube des années 1970, accompagne autant qu'elle promeut un mouvement de régénération de l'institution muséale (Desvallées 1992, 1994). On sait l'histoire de ce courant, à l'époque révolutionnaire et présenté comme la seule alternative crédible aux musées de cette époque, alors en crise (Clair 1971;O’Doherthy 1972; Varine-Bohan 1975). Les noms de Georges Henri Rivière et d'Hugues de
Varine, qui y sont associés, ont contribué à donner à ce mouvement une renommée internationale... du moins dans les pays latins. Car s'il existe un certain nombre de pionniers américains dont se réclame la nouvelle muséologie -comme John Coton Dana ou Theodor Low et plus récemment John Kinard- c'est essentiellement à partir de la France que le courant se dissémine, et d'abord au Québec, en Espagne, au Portugal et en Amérique latine. S'il existe ainsi, au début du $\mathrm{XXI}^{\mathrm{e}}$ siècle, quelques centaines d'écomusées (Maggi 2002), ceux-ci sont évidemment nettement moins nombreux et célèbres que les prestigieux édifices qui ont éclos aux quatre coins du globe et dont le Musée Guggenheim de Bilbao constituait, en quelque sorte, l'étendard.

A l'époque de la crise des musées, pourtant, le monde des musées et la muséologie se présentent déjà réellement face à des alternatives divergentes. Tandis que le musée de la fin des années 1960 est critiqué pour son obsolescence, plusieurs alternatives s'offrent au secteur: la nouvelle muséologie propose une voie résolument orientée vers les citoyens en renversant, en quelque sorte, la priorité du rapport à l'objet entretenue auparavant. A la même époque, la muséologie de l'Est, en revanche, estime que la crise muséale constitue la résultante d'un trop faible investissement scientifique dans la muséologie: c'est parce que celle-ci n'aurait pas encore accédé au statut de science qu'elle ne serait pas parvenue à répondre de manière cohérente aux enjeux actuels. On sait la fortune de chacune de ces deux propositions, progressivement abandonnées au bénéfice d'un courant plus directement lié à l'architecture muséale (qui a pu être qualifié de spectaculaire muséal (Mairesse 2002).

\section{Muséologie sociale, muséologie critique}

Forcément, le caractère plus largement participatif et communautaire du musée, tel qu'il est annoncé, laisse penser que la muséologie pourrait se présenter davantage, durant les prochaines années, sous un angle social. Nombre d'indices, à cet égard, montrent le redéploiement de ce courant, à commencer par le lancement des cadernos de sociomuseologia, institués dans la filiation de la nouvelle muséologie et notamment des écrits de Pierre Mayrand (2009). La muséologie sociale, ou sociomuséologie, positionne, à la suite de la nouvelle muséologie, le visiteur/acteur au cœur d'un processus visant à utiliser la mémoire 
collective ou le patrimoine comme un outil de travail et de développement. On peut rattacher à ce courant nombre de contributions anglo-saxonnes présentant le travail social à l'intérieur du musée (Silverman 2010), l'inclusion sociale (Sandell 2003) ou le travail collaboratif (Simon 2010). Sans doute le terme de muséologie sociale s'avère-t-il plus directement compréhensible que celui, parfois ambigu, de nouvelle muséologie, dont le pendant «new museology», utilisé notamment par Peter Vergo (1989), avait très largement suscité la confusion. Cette dernière proposition, fondée dans le courant des cultural studies, adoptait une approche moins fondée sur le développement social que la déconstruction des dispositifs muséaux au sein de la société, à la lumière d'un Foucault et de la French Theory. Le terme de muséologie critique (critical museology) qui, depuis quelques années, s'est répandu et rassemble aussi bien ce type d'approches que, de manière plus générale, la perspective marxiste (d'un Adorno et Horkheimer), définit probablement mieux le contenu de ces analyses, essentiellement universitaires (Lorente 2012b). Depuis quelques années, ces approches critiques, d'abord fondées sur des disciplines classiques (histoire, sciences politiques, ethnologie), se sont de plus en plus emparées de nouvelles perspectives d'analyse issues des études postcoloniales ou des études sur le genre (Simpson 1996; Chambers et al. 2014; Levin 2010).

Les développements actuels de la muséologie semblent ainsi prendre des directions quelque peu différentes de la muséologie «traditionnelle», fondée sur l'histoire des musées, leur organisation (collections, expositions, architecture) et les études de public. Se dégageraient ainsi deux voies particulières: l'une plutôt sociale, fondée essentiellement sur les liens entre musées, sociétés et communautés (community), dans une visée clairement participative, à partir d'expériences concrètes et des acteurs cherchant à théoriser leur démarche (comme Nina Simon, au sein du Musée de Santa Cruz); l'autre plutôt critique mais aussi plus concentrée à l'intérieur du monde universitaire, envisagée à partir de l'analyse sociopolitique du musée, mais aussi de nouvelles perspectives comme le genre ou les études coloniales. Il existe quelques tentatives de rapprochement entre ces deux propositions (Dewdney, Dibosa y Walsh 2013), lesquelles apparaissent néanmoins comme relativement divergentes.

\section{L'avenir anglo-saxon de la muséologie}

A ce constat, il convient par ailleurs d'ajouter l'influence de plus en plus prépondérante du modèle anglo-saxon au sein du champ muséal ${ }^{5}$. La chute du mur de Berlin et l'implosion du bloc soviétique marquant la désagrégation du système socialiste entraînent le développement accéléré des politiques de marché libérales, telles que mises en place dans les pays anglo-saxons. On observe ainsi, dans l'ensemble du monde occidental, une diminution croissante des politiques publiques afin de «libérer» les entraves du marché (Harvey 2005). Le phénomène est observable dans le monde des musées, contraint de s'adapter à cette nouvelle réalité. En l'espace d'une génération, le secteur des musées va donc lui aussi subir un changement radical... au bénéfice, certainement, du bloc le plus fort sur le plan économique. Si, au seuil des années 1980, le monde de la muséologie présente ainsi encore un ensemble de modes de pensée relativement hétérogène (anglo-saxonne, de l'Est, latine, nouvelle muséologie), cet ensemble évolue rapidement à partir des années 1990. Importance de la langue? Développement économique rapide? Transformation du système universitaire? Il est difficile de ne retenir qu'un seul facteur explicatif pour évoquer l'importance du changement qui s'opère notamment en Grande-Bretagne, mais quoiqu'il en soit, il apparaît que les publications de $m u$ seum studies, à travers les éditions Routledge notamment, de même que celles de l'Université de Leicester, connaissent à cette époque une notoriété mondiale. En l'espace de quelques années, des dizaines de publications sortent des presses de ces maisons d'édition, de nombreux ouvrages s'imposant rapidement comme des classiques du genre ${ }^{6}$. Il est vrai que l'anglais international, langue de l'empire britannique, s'est largement imposé à travers le monde comme lingua franca, véhicule des échanges internationaux, au détriment du français, de l'espagnol ou du russe. On ne peut qu'apprécier la possibilité pour tout un chacun de communiquer avec le reste du monde. Le choix d'une langue commune, dans cette perspective, s'avère pour le moins décisif. 


\section{Des approches largement différentes}

Le passage d'une langue à l'autre n'est pas sans influence sur l'expression d'une pensée, voire sur la formation d'une discipline. Dès la fin des années 1950 , la linguistique se penche sur la question de la relativité de son approche (avec Edward Sapir et Benjamin Whorf): la manière dont nous percevrions le monde dépendrait (en partie) de la structure de notre langage (Lucy 1992). Il est évidemment complexe de saisir à quel point le langage, s'il est constitutif de notre culture, peut déterminer notre manière d'appréhender et d'exprimer une situation. Tout individu amené à transposer sa pensée dans une autre langue comprend intuitivement les écarts entre l'un et l'autre univers linguistiques. Sur le champ muséal, le simple choix d'un terme, muséologie, vs museum studies, renseigne déjà sur des orientations largement différentes en matière de contenu des enseignements et des modes de réflexion (Gómez Martínez 2006), et si des termes comme muséal ou muséalisation apparaissent relativement familier à un public latin, ils sonnent pour le moins étrangement dans de nombreuses autres langues.

A ce premier écart-linguistique-il convient de souligner la différence (voire la divergence) des formations philosophiques entre pays, conditionnant la réflexion épistémologique (par exemple la manière de concevoir la muséologie et ses méthodes). Il est important de souligner combien, au sein du monde occidental, on n'enseigne pas et on ne se réfère pas, par exemple, aux mêmes philosophes... Tandis qu'en France et en Allemagne, le cartésianisme (Descartes) et le rationalisme (Leibniz) jouissent d'une position encore centrale, en Grande Bretagne, c'est l'empirisme (Bacon, Locke, Hume) qui s'impose, présentant l'expérience à l'origine du système des connaissances, contrairement au rationalisme se fondant sur la raison discursive. A ces différences, il convient d'ajouter le développement, à partir du $\mathrm{XX}^{\mathrm{e}}$ siècle, du pragmatisme américain (Peirce et Dewey), centré sur l'action et ses conséquences. De telles divergences, dans les formations, permettent de mieux comprendre les réticences ou parfois l'hostilité qu'éprouvent certains penseurs anglo-saxons pour la prose de leurs homologues latins ou germains - et vice-versa. On retrouve ainsi, par exemple, une réelle méfiance pour Hegel et son système de connaissance chez l'historien de l'art Gombrich (1992), et une hostilité affichée pour la muséologie scientifique de l'Est, chez le muséologue britannique Kenneth Hud- son (1997). La volonté de ne rechercher dans la muséologie que des réponses à des questions pratiques fondées sur l'expérience, telle qu'on la retrouve affirmée de manière explicite chez un Burcaw (et totalement dénigrée par un Stránský), semblent ainsi s'opposer à la spéculation intellectuelle de la muséologie théorique ( Burcaw 1981).

\section{La muséologie à la croisée des chemins}

Le monde des musées apparaît ainsi confronté non seulement à une crise de croissance (et à la croissance de la crise), mais également à des transformations importantes de la société (démographie, technologies numériques, économie et rôle de l'Etat); il en va forcément de même pour la muséologie: l'un ne peut manquer d'affecter l'autre. Les études prospectives sur le musée présentent ce dernier comme destiné à jouer un rôle plus social, à l'écoute de ses publics ou, de manière générale de ses parties prenantes (stakeholders): moins d'espaces pour les collections permanentes, plus pour les événements, les actions en lien avec l'actualité, les rencontres, les débats ou les performances. Le modèle du musée comme centre de la vie sociale, conduit et organisé par l'ensemble des membres de la communauté, constitue une figure ancienne (Cameron 1971). C'est cette vision que l'on retrouve au centre du paradigme animant la nouvelle muséologie, puis la muséologie sociale. Une telle vision ne s'oppose pas vraiment à celle, plus théorique, de la muséologie critique, reposant sur l'étude de l'influence des cadres de la pensée (économie, genre, colonialisme,...) sur le fonctionnement de l'institution. En quelque sorte, l'un et l'autre mode de pensée sont sans doute poursuivis par des acteurs différents, largement impliqués dans le travail muséal participatif, pour ce qui concerne la muséologie sociale, plus directement engagés dans une logique académique pour la muséologie critique. Pour autant, on ne peut dire qu'ils s'excluent mutuellement.

Si l'on peut parler de croisée des chemins, c'est moins parce que ces deux démarches sont soutenues par des impératifs différents (des enjeux pratiques dans le premier cas et plus académiques dans le second), que parce qu'il convient de reconnaître, à travers cette évolution, la constitution d'un modèle global de plus en plus dominant à travers le monde. Un modèle fondé sur une approche essentiellement anglo-saxonne, privilé- 
giant la littérature ainsi que les acteurs s'insérant dans les cadres de cette pensée. Elles sont loin, les conceptions théoriques de l'Est visant à constituer la muséologie comme une science particulière et indépendante, fondée sur un objet plus large que le musée. Elles sont tout aussi éloignées, les références latines et notamment francophones utilisées un peu partout à travers le monde, à l'initiative de l'ICOM (Rivière, Varine, Monreal). La diversité de pensée et l'équilibre des différents courants au sein de la muséologie, que l'on pouvait encore apprécier il y a trois décennies, apparaît comme de plus en plus menacée en regard de la production certes pas vraiment monolithique, mais certainement mainstream (Martel 2010), de la muséologie actuelle - bien qu'il existe, faut-il le remarquer, une littérature de plus en plus abondante dans les pays latins (relayée par les éditions de 1'Harmattan, Arman Colin, Trea, la Documentation française, etc.). Il est vrai que ce courant général suscite largement l'intérêt des publics -étudiants, académiques ou professionnels- à qui il s'adresse. Cette perspective future de la muséologie est-elle pour autant inéluctable? Certes, les particularismes subsistent et subsisteront encore dans les années à venir : il ne devrait pas être nécessaire de faire entrer la muséologie dans les objets de la Convention de 2005 pour la protection et la promotion de la diversité des expressions culturelles... Il n'en reste pas moins qu'une réflexion s'impose: la perspective mainstream, pour intéressante qu'elle soit, n'oblitère-t-elle pas un certain nombre de pistes de recherche liées au champ muséal? La perspective théorique, dominante dans l'ancienne approche des pays de l'Est mais encore largement présente dans la littérature francophone, hispanophone ou lusophone ${ }^{7}$, n'est elle pas négligée? De manière plus générale, par-delà les community museums et les musées superstars, quel pourrait être l' «objet» de la muséologie dans les années à venir? Si «l'étude d'une relation spécifique de l'homme à la réalité» ne suffit probablement plus à susciter la captatio benevolentice des chercheurs ou des étudiants, de nouvelles voies alternatives visant à explorer le champ muséal ne se dégagent-elles pas?
De tels questionnements reviennent à envisager l'analyse prospective au niveau non plus du musée, mais de la muséologie elle-même, comme champ de recherche et d'enseignement. Ce travail n'a, à ma connaissance, pas encore été vraiment réalisé, mais son ampleur dépasse largement le cadre de cette contribution. Il s'agit en effet de ne pas seulement se concentrer sur la possible évolution des musées, mais aussi sur celle des universités (et de manière plus générale, du système d'apprentissage) et de partenaires relativement proches du musée, comme les bibliothèques ou les centres d'archives, pour lesquels les travaux de prospective sont tout aussi importants ${ }^{8}$. Les mondes de la recherche et de l'enseignement, eux-mêmes sont en train de connaître d'importantes modifications - dans le sillage du déploiement de la logique néolibérale et de la mondialisation. Les changements démographiques, le développement des technologies numériques et la diminution des ressources publiques constituent, comme pour les musées, des constantes pour l'ensemble de ces institutions. La mobilité des enseignants ou des chercheurs, mais aussi celle des étudiants, la compétition de plus en plus forte entre les établissements, l'accroissement exponentiel des ressources d'informations, à l'intérieur des systèmes institutionnels (universités, bibliothèques et musées) autant qu'à l'extérieur, constituent un univers tout aussi mouvant pour la muséologie, amenée à accompagner les changements du système muséal, mais aussi ceux des autres institutions patrimoniales, académiques et éducatives.

Ces inconnues face au futur laissent l'opportunité d'imaginer d'autres chemins que ceux évoqués plus haut et présentés, a priori, comme pratiquement inéluctables. Mais ces chemins alternatifs impliquent d'ajouter, à la réflexion sur le champ muséal, celle sur les nouvelles pratiques de recherche et d'enseignement ou, de manière plus globale, sur les nouvelles compétences souhaitées 9 . C'est à ce niveau que des perspectives potentiellement différentes, pour la muséologie mais sans doute aussi pour le musée, pourraient se dessiner. Un tel travail d'analyse, de réflexion et de propositions s'annonce pour le moins complexe, mais ses résultats s'avèrent cruciaux pour le développement du champ de la muséologie. 


\section{NoTeS}

1. Je reprends, dans cette première partie, des idées exprimées dans Mairesse, F. (2013): Eléments de prospective muséale. La lettre de l'OCIM, 150: 9-14 et L'économique et/ou le social? Quel rôle futur pour le musée? Culture et inclusion sociale (A. Barrere, F. Mairesse) Paris, L'Harmattan, à paraître. C'est dans ce dernier article que l'analyse détaillée des rapports de prospective est présentée.

2. Ces trois rapports sont disponibles sur le site du Centre pour l'avenir des musées: http://www.aam-us.org/ resources/center-for-the-future-of-museums.

3. Museums Association (2012): Museums 2020 Discussion Paper. Museums Association, London. Disponible sur Internet: [URL: http://www.museumsassociation.org/download?id=806530; Nederlandse Museumvereniging].

(2010): Agenda 2026. Study on the Future of the Dutch Museum Sector. Amsterdam. Disponible sur Internet: [URL: http://www.museumvereniging.nl/LinkClick.aspx?fileticket=jEC-ghnfc3A\%3D\&tabid=244. La consultation à partir de Museums 2020 a donné lieu à une synthèse présentant la vision de la MA: Museums Change Lives. Disponible sur Internet: [URL:http://www.museumsassociation.org/download?id=1001738]. Consultation février 2014.

4. Par ailleurs, si presque tous les rapports sont structurés à partir des principaux facteurs de changement, celle utilisée par la Museums Association se fonde sur un scénario du futur, construit à partir de ces transformations.

5. Je reprends ici, dans ce paragraphe, certaines idées de mon article Le musée inclusif et la muséologie mondialisée/ O museu inclusivo e a museologia mundializada. Icofom-Lam, 21 encuentro regional, Termos e conceptos de museologia: museu inclusivo, interculturalidade et patrimonio integral, Rio/Petropolis, 2012: $17-52$.

6. Notamment à travers l'œuvre de Pearce et de Hooper Greenhill, ainsi que de la série Leicester reader in Museum Studies.

7. On songe notamment aux écrits de Bernard Deloche, mais aussi aux travaux d'Icofom-Lam, en Amérique latine. Voir par exemple Deloche, B. (2003): El museo virtual, Trea, Gijón.

8. Voir par exemple King, G.; Maya, S. (2013): The Troubled Future of Colleges and Universities (with comments from five scholar-administrators), PS: Political Science and Politics 46 (1): 81-113. Disponible sur Internet: http://j.mp/U82gj2; Ernst and Young (2012): University of the Future. A thousand year old industry on the cusp of profound change. Disponible sur Internet: [URL:http://www.ey.com/Publication/ vwLUAssets/University of the future/\$FILE/University of the future 2012.pdf];Curtis, G. et al. (2011): Academic libraries of the Future. Scenarios beyond 2020. British Library, London. Disponible sur Internet: [http://www.sconul.ac.uk/sites/default/files/documents/Scenarios_beyond_2020_ReportWV.pdf].

9. Voir par exemple Davies, A.; Fidler, D.; Gorbis, M. (2011): Future work skills. Institute for the Future for the University of Phoenix Research Institute, Palo Alto, California. Disponible sur Internet:[URL: http:// www.iftf.org/uploads/media/SR-1382A_UPRI_future_work_skills_sm.pdf]. Consultation février 2014.

\section{RÉFÉRENCES BibLIOGRAPHIQUES}

Black, G. (2012): Transforming Museums in the Twenty-first Century, Routledge, London.

BRown Goode, G.(1896): The principles of museum administration, Report of Proceedings with the papers read at the sixth annual general meeting, held in Newcastle-upon-tyne, July 23rd-26th, Dulau, London: 69-148.

Burcaw, G.E. (1981): Réflexions sur MuWop no 1. MuWop/Do Tram, 2: 87.

CAMERon, D. (1971): Museum, a temple or a forum. Curator, 14, march: 11-24.

Chambers, I. et al. (2014): The postcolonial Museum, The Arts of Memory and the Pressures of History, Ashgate, London.

Clair, J. (1971): La fin des musées? Chroniques de l'Art vivant, 24, oct.: 5.

DeLoche, B. (2003): El museo virtual, Trea, Gijón. 
Desvallées, A. (1992 y 1994): Vagues. Une anthologie de la nouvelle muséologie, Ed. W. et M.N.E.S., Màcon, 2 vol.

Desvallées, A.; Dibosa, D.; Walsh, V. (2013): Post-critical Museology. Theory and Practice in the Art Museum, Routledge, London.

Freyre, B.; MeIEr, S. (2006): The Economics of Museums.., Handbook of the Economics of Art and Culture (Ginsburigh, V., Throsby, D., eds.), Elsevier, Amsterdam vol. 1: 1017-1050.

GomBrich, F. (1992): En quête de l'histoire culturelle, Gérard Montfort, Paris.

Gómez Martínez, J. (2006): Dos museologías. Las tradiciones anglosajona y mediterránea: diferencias y contactos, Trea, Gijón.

Harvey, D. (2005): A brief History of Neoliberalism. Oxford University Press, Oxford. Hudson, K. (1997): Who are the 'museologists' and for whose benefit do they exist? Museology for Tomorrow's World (Stránský Z. Z., ed.), Müller-Straten, München: 102-110.

Levin, A. (ed.) (2010): Gender, Sexuality and Museums. A Routledge Reader, Routledge, London.

LORENTE, J.P. (2012A): The development of museum studies in universities: from technical training to critical museology. Museum management and curatorship, 27:3: 237-252.

Lorente, J.P. (2012B): Manual de historia de la museología, Trea, Gijón.

Lucy, JA. (1992): Language Diversity and Thought: A Reformulation of the Linguistic Relativity Hypothesis. Cambridge University Press, Cambridge.

MagGi, M. (2002): Ecomusei. Guida europea, Umberto Allemandi; C., Ires Piemonte.

MaIREsSe, F. (2002): Le musée, temple spectaculaire, Presses Universitaires de Lyon, Lyon.

Mairesse, F.; Desvallés, A. (2011): Muséologie. Dictionnaire encyclopédique de muséologie (Desvallées, A.; Mairesse, F., dir.), Armand Colin, París: 343-384.

Martel, F. (2010): Mainstream. Enquête sur cette culture qui plaît à tout le monde, Flammarion, Paris.

Mayrand, P. (2009): Paroles de Jonas. Essais de terminologie augmentés des chroniques d'un altermuséologue. Cadernos de Sociomuseologia, 31: 19. [URL http://revistas.ulusofona.pt/index.php/cadernasociomuseologia/issue/archive]. Consultation 11/6/2014.

Mensch, P.; L. Van (2011): New Trends in Museology, Museum of Recent History, Celsje.

O’Doherty, B. (ed.) (1972): Museums in Crisis, Braziller, New York.

Pettersson, S. et al. (ed.) (2010): Encouraging collections mobility - A way Forward for Museums in Europe, Helsinki, Finnish National Gallery, Amsterdam, Erfgoed Nederland et Berlin, Staatliche Museen.

Rivière, G. H. (1989): La Muséologie selon Georges Henri Rivière:Cours de muséologie/Textes et témoignages, Dunod-Bordas, París.

SANDELl, R. (1998): Museums as agents of social inclusion. Museum Management and curatorship, 17, 4: 401-418.

SAndell, R. (ed.) (2002): Museums, society, inequality, Routledge, London.

SANDELL, R. (2003): Social inclusion, the museum and the dynamics of sectoral change. Museum and Society $1(1): 45-62$.

Silverman, L. H. (2010): The Social Work of Museums, Routledge, London.

Simon, N. (2010): The participatory museum, Museum, s.1., 2.0. [URL http://www.participatorymuseum. org. Consultation 5/6/2014.

Simpson, M. (1996): Making representations. Museums in the Post-colonial Era. Routledge, London.

VARIne-Bohan, H. DE (1975): Les musées dans le monde, Grammont-Laffont, Lausanne.

VERGo, P. (ed.) (1989): The New Museology, Reaktion books, London. 